\title{
Suporte da Gestão do Conhecimento em práticas de Ecoinovação
}

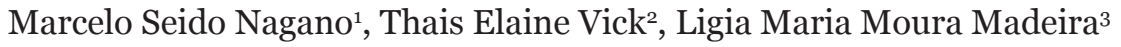 \\ drnagano@usp.br, thavick@sc.usp.br, ligiamadeira@usp.br \\ 1,2,3Universidade de São Paulo, Escola de Engenharia de São Carlos, Departamento de Engenharia de \\ Produção, Av. Trabalhador Sãocarlense, 400 - 13566-590, São Carlos - SP, Brasil
}

DOI: 10.17013/risti.22.37-56

\begin{abstract}
Resumo: $\mathrm{O}$ artigo tem como propósito analisar o relacionamento entre as práticas de ecoinovação e gestão do conhecimento. Ênfase é dada ao desenvolvimento de ferramentas para o desenho ambiental, uma vez que estas representam uma forma de integrar recursos de conhecimento ao processo de desenvolvimento de produto. Sabe-se que o comprometimento da alta gestão é imprescindível na mudança organizacional para a implementação de sistemas de gestão ambiental. Outrossim, a gestão deve fomentar a criação de conhecimento. Como forma de demonstrar a relação que é objeto de pesquisa, são estudados o Standard Design Process Form e o Diagrama de Product Ideas Tree. Tais metodologias foram selecionadas por caracterizarem de forma elucidativa o processo de geração de idéias dentro da ecoinovação. Além das relações destacadas entre as práticas de gestão do conhecimento e ecoinovação, um conjunto de quatro proposições é elencado no intuito de ressaltar os resultados encontrados na discussão.
\end{abstract}

Palavras-chave: Ecoinovação; Gestão do Conhecimento; Criação de Conhecimento; Gestão Ambiental.

\section{Support of Knowledge Management in Eco-innovation practices}

\begin{abstract}
The article aims to analyze the relationship between the practices of ecoinnovation and knowledge management. Emphasis is given to the development of tools for environmental design, since they represent a way of integrating knowledge resources to the process of product development. It is known that the commitment of top management is essential for the implementation of environmental management systems. Likewise, management should promote knowledge creation. As a way to demonstrate the relationship that is the subject of research, the Standard Design Process Form and the Product Ideas Tree diagram are studied. These methodologies were selected in order to characterize and enlighten the process of generating ideas in the eco-innovation. In addition to the relationships found between the knowledge management and eco-innovation practices, a set of four propositions is listed in order to emphasize the results found in the discussion.
\end{abstract}

Keywords: Eco-innovation; Knowledge Management; Knowledge Creation; Environmental Management. 


\section{Introdução}

Uma das temáticas mais relevantes da atualidade e que merece uma atenção especial é a questão ambiental, uma vez que esta envolve tanto aspectos relacionados ao ambiente e a indústria, como métodos de produção mais limpos, bem como a demanda dos consumidores por produtos ambientalmente mais corretos.

A ecoinovação depende da atribuição de significado e valor ao conhecimento ambiental (Huang et al., 2009; Huang et al., 2010; Vicente-Molina et al., 2013; Wernick, 2003). Partindo desse princípio, sabe-se que a capacidade de desenho ambiental (i.e. processos e produtos) é derivada dos recursos organizacionais e das rotinas que facilitam as atividades exploratórias e que ajudam a descobrir e a criar novos conhecimentos ambientais.

A capacidade de incorporar as preocupações ambientais no processo de desenvolvimento de produtos campeia atualmente como um dos requisitos de maior demanda pelas empresas (Blomquist e Sandstrom, 2004; Dangelico e Pujari, 2010; Kobayaschi, 2011; Pujari, 2006; Scarpellini et al., 2012). À vista disso, sabe-se que a capacidade de desenho ambiental deriva do conhecimento especializado com relação à:

- Impactos ambientais;

- Recursos de conhecimento internos e externos da organização, coordenados com a equipe de desenvolvimento de produtos.

Os recursos do conhecimento são informações e conhecimento especializado que residem nos indivíduos, nos grupos, e nos artefatos técnicos.

Dessarte, ênfase deve ser dada ao desenvolvimento de ferramentas de desenho ambiental, uma vez que elas representam um modo de integrar os recursos de conhecimento ao processo de desenvolvimento de produtos. E é exatamente neste ponto que reside a proposta desse artigo. A capacidade de desenho ambiental é, portanto, o resultado de um processo de aprendizagem (Aravind, 2012; Elmquist e Segrestin, 2009; Freixo \& Rocha 2014, Gavronski et al., 2012; Hallstedt et al., 2013; Jones et al., 2001; Lenox e Ehrenfeld, 1997; Malmborg, 2007; Quist e Tukker, 2013; Siebenhuner e Arnold, 2007; Sokolovic, 2012).

A pesquisa em ecoinovação está ainda em sua fase inicial (Andersen, 2008) e são poucas as pesquisas em inovação que trabalham com as questões ambientais. A Europa é o continente que se destaca com relação à estratégia da ecoinovação, apesar de serem poucos os casos. No continente europeu, há uma disparidade entre o investimento privado e o público em pesquisa e desenvolvimento (P\&D), em milhões de dólares, voltados para esta prática. No entanto, os investimentos privados são sobrepujantes contra os públicos (Scarpellini et al., 2012).

Ademais, existe uma lacuna que se relaciona aos fatores sócio-culturais, ou ainda a cultura organizacional e recursos humanos e a ligação com as inovações ambientais (Baumgartner e Zielowski, 2007; Jabbour et al., 2011; Reid e Miedzinski, 2008). Segundo Tidd (2001), os dois ingredientes básicos para o bom desempenho da atividade 
inovadora são os recursos técnicos (pessoas, infraestrutura, conhecimento e capital), e as competências organizacionais para gerenciá-los. Ainda, a P\&D é particularmente um objeto de estudo interessante acerca do greening das empresas (Blomquist e Sandstrom, 2004).

Tendo isso em vista, este artigo possui uma característica inovadora, uma vez que aborda o conceito de ecoinovação estabelecendo um forte relacionamento com a gestão do conhecimento (GC). Cabe salientar que a GC é vista como uma estratégia ou ferramenta para a implementação de certificações ambientais como a ISO 14001, por exemplo. Esta relação será estabelecida no decorrer das próximas seções do artigo. A partir desta ligação entre as duas práticas, podem-se observar relações de aprendizagem por meio de algumas técnicas ou métodos.

\section{Metodologia}

O artigo apresenta uma pesquisa bibliográfica, segundo seus procedimentos. Busca a exposição de conhecimentos para uma nova tomada de posição, auxiliando na compreensão do problema a partir da análise de contribuições científicas e através de duas dimensões consecutivas: 1) fundamentação (revisão de literatura) e 2) construto teórico, ou seja, contribuições deste trabalho para o campo teórico de conhecimento.

Inicialmente, o levantamento sistemático da literatura especializada ocorreu mediante uma busca inicial em bases de dados (Web of Science e SciELO) a partir do cruzamento de palavras-chaves para o levantamento de periódicos relacionados aos temas, no período que compreende os anos de 1990 a 2013.

Obteve-se assim uma amostra inicial com aproximadamente 500 artigos, que abordaram um dos grandes temas em estudo: Ecoinovação, Gestão do Conhecimento, Conhecimento Ambiental e Sustentabilidade.

Dos 500 trabalhos, apenas 80 preencheram os requisitos fixados para a revisão sistemática, uma vez que os 80 artigos selecionados trataram (de forma não explícita), de ecoinovação e suas possíveis relações com a gestão do conhecimento. A Tabela 1 a seguir traz o levantamento final dos campos que o trabalho aborda, bem como os autores que os representam, no intuito de auxiliar o entendimento dos temas-chave do artigo:

Tem-se como questão de pesquisa: Como as práticas de Ecoinovação e Gestão do Conhecimento estão relacionadas? Foram selecionadas para ilustrar e exemplificar a relação proposta no artigo duas ferramentas: o Standard Design Process Form - SDPF (INNS, 1994) e o Diagrama de PIT (JONES et al., 2001). Tais metodologias dão ênfase ao desenho sustentável dos produtos (DSP) no processo de desenvolvimento de produtos (PDP), possibilitando uma visão de onde a 'geração de idéias' ocorre (Johansson e Magnusson, 1998; Jones et al., 2001).

O presente artigo também oferece um conjunto de proposições que levam ao estabelecimento de uma relação benéfica entre a GC e a ecoinovação, para apoiála a fim de se obter uma eficaz gestão do processo de desenvolvimento de produtos ambientalmente mais sustentáveis. 


\begin{tabular}{|c|c|c|c|c|c|}
\hline Anos & Autores & $\begin{array}{l}\text { Inovação } \\
\text { Ecoinovação } \\
\text { \& PDP }\end{array}$ & $\begin{array}{l}\text { Conhecimento e } \\
\text { Gestão }\end{array}$ & $\begin{array}{l}\text { Conhecimento } \\
\text { Ambiental }\end{array}$ & Sustentabilidade \\
\hline 1990 & $\begin{array}{l}\text { 2.1Cohen e Levinthal } \\
\text { 2.2Prahalad e Hamel }\end{array}$ & & $\begin{array}{l}2.1 \\
2.2\end{array}$ & & \\
\hline 1994 & $\begin{array}{l}\text { 3.1Inns } \\
\text { 3.2Iansiti e Clark }\end{array}$ & $\begin{array}{l}3.1 \\
3.2 \\
\end{array}$ & & & \\
\hline 1995 & $\begin{array}{l}\text { 4.1 Hart } \\
\text { 4.2Nonaka e Takeuchi } \\
\text { 4.3Porter e Van der } \\
\text { Linde } \\
\text { 4.4Shrivastava }\end{array}$ & $\begin{array}{l}4 \cdot 3 \\
4 \cdot 4\end{array}$ & 4.2 & 4.3 & $\begin{array}{l}4.1 \\
4.3 \\
4.4 \\
\end{array}$ \\
\hline 1996 & 5.1Inkpen & & 5.1 & & \\
\hline 1997 & 6.1Lenox e Ehrenfeld & 6.1 & & 6.1 & 6.1 \\
\hline 1998 & $\begin{array}{l}\text { 7.1Amabile } \\
\text { 7.2 Von Krogh } \\
\text { 7.3Leonard e Sensiper } \\
\text { 7.4Johansson }\end{array}$ & $\begin{array}{l}7 \cdot 3 \\
7 \cdot 4\end{array}$ & $\begin{array}{l}7.1 \\
7.2\end{array}$ & & 7.4 \\
\hline 1999 & $\begin{array}{l}\text { /8.1Johannessen et al } \\
\text { 8.2Klassen e } \\
\text { Whybark } \\
\text { 8.3Noci e Verganti }\end{array}$ & $\begin{array}{l}8.2 \\
8.3\end{array}$ & 8.1 & & $\begin{array}{l}8.2 \\
8.3\end{array}$ \\
\hline 2000 & $\begin{array}{l}\text { 9.1Nonaka e Toyama } \\
\text { 9.2Rennings }\end{array}$ & 9.2 & 9.1 & & 9.2 \\
\hline 2001 & $\begin{array}{l}\text { 10.1El Sawy et al } \\
\text { 10.2Jones et.al } \\
\text { 10.3Jones et.al } \\
\text { 10.4Tidd et al }\end{array}$ & $\begin{array}{l}10.1 \\
10.2 \\
10.3 \\
10.4\end{array}$ & $\begin{array}{l}10.1 \\
10.4\end{array}$ & $\begin{array}{l}10.2 \\
10.3\end{array}$ & $\begin{array}{l}10.2 \\
10.3\end{array}$ \\
\hline 2002 & $\begin{array}{l}\text { 11.1Boiral } \\
\text { 11.2Silva }\end{array}$ & 11.1 & 11.2 & 11.1 & 11.1 \\
\hline 2003 & $\begin{array}{l}\text { 12.1Brannback } \\
\text { 12.2Melnyk et al } \\
\text { 12.3Mitri } \\
\text { 12.4Nonaka e Toyama } \\
\text { 12.5Wernick }\end{array}$ & $\begin{array}{l}12.1 \\
12.2\end{array}$ & $\begin{array}{l}12.1 \\
12.3 \\
12.4\end{array}$ & $\begin{array}{l}12.2 \\
12.5\end{array}$ & $\begin{array}{l}12.2 \\
12.5\end{array}$ \\
\hline 2004 & $\begin{array}{l}\text { 13.1Blomquist e } \\
\text { Sandstrom }\end{array}$ & 13.1 & 13.1 & 13.1 & 13.1 \\
\hline 2005 & 14.1Lee e Rhee & 14.1 & & 14.1 & 14.1 \\
\hline 2006 & $\begin{array}{l}\text { 15.1Kobayaschi } \\
\text { 15.2Pujari }\end{array}$ & $\begin{array}{l}15.1 \\
15.2\end{array}$ & & & $\begin{array}{l}15.1 \\
15.2\end{array}$ \\
\hline
\end{tabular}




\begin{tabular}{|c|c|c|c|c|c|}
\hline 2007 & $\begin{array}{l}\text { 16.1Baumgartner e } \\
\text { Zielowsk } \\
\text { 16.2Falk e Ryan } \\
\text { 16.3Hellstrom } \\
\text { 16.4Kleef e Roome } \\
\text { 16.5Siebenhuner e } \\
\text { Arnold }\end{array}$ & $\begin{array}{l}16.2 \\
16.3 \\
16.4\end{array}$ & $\begin{array}{r}16.1 \\
16.5\end{array}$ & 16.5 & $\begin{array}{l}16.1 \\
16.2 \\
16.3 \\
16.4 \\
16.5\end{array}$ \\
\hline 2008 & $\begin{array}{l}\text { 17.1Andersen } \\
\text { 17.2Horbach et al } \\
\text { 17.3 Kemp e Volpi } \\
\text { 17.4Reid e Miedzinski } \\
\text { 17.5Jabbour et al }\end{array}$ & $\begin{array}{l}17.1 \\
17.2 \\
17 \cdot 3 \\
17.4\end{array}$ & 17.5 & 17.1 & $\begin{array}{l}17.1 \\
17.2 \\
17.3 \\
17.4 \\
17.5 \\
\end{array}$ \\
\hline 2009 & $\begin{array}{l}\text { 18.1Huang et al } \\
\text { 18.2Kammerer } \\
\text { 18.3Oliveira e } \\
\text { Pinheiro } \\
\text { 18.4Elmquist e } \\
\text { Segrestin }\end{array}$ & $\begin{array}{l}18.1 \\
18.2 \\
18.3\end{array}$ & $\begin{array}{l}18.3 \\
18.4\end{array}$ & $\begin{array}{l}18.1 \\
18.2 \\
18.3 \\
18.4\end{array}$ & $\begin{array}{l}18.1 \\
18.2 \\
18.3 \\
18.4\end{array}$ \\
\hline 2010 & $\begin{array}{l}\text { 19.1Carrillo et al } \\
\text { 19.2Huang e Wu } \\
\text { 19.3Tseng } \\
\text { 19.4Huang et al } \\
\text { 19.5 Pujari }\end{array}$ & $\begin{array}{l}19.1 \\
19.2 \\
19.3 \\
19.4 \\
19.5 \\
\end{array}$ & & $\begin{array}{l}19.2 \\
19.3 \\
19.4 \\
19.5\end{array}$ & $\begin{array}{l}19.1 \\
19.2 \\
19.3 \\
19.4 \\
19.5 \\
\end{array}$ \\
\hline 2011 & $\begin{array}{l}\text { 20.1Jabbour et al } \\
\text { 20.2Kobayaschi et al } \\
\text { 20.3Santolaria et al } \\
\text { 20.4Segarra-Oña et al }\end{array}$ & $\begin{array}{l}20.2 \\
20.3 \\
20.4 \\
\end{array}$ & 20.1 & 20.1 & $\begin{array}{l}20.1 \\
20.2 \\
20.3 \\
20.4 \\
\end{array}$ \\
\hline 2012 & $\begin{array}{l}\text { 21.1Aravind } \\
\text { 21.2Bloodgood } \\
\text { 21.3Carrillo et al } \\
\text { 21.4Carruthers } \\
\text { 21.5Doran e Ryan } \\
\text { 21.6Gavronski } \\
\text { 21.7Horbach et al } \\
\text { 21.8Jabbour et al } \\
\text { 21.9Kesidou } \\
\text { 21.10Paraschiv et al } \\
\text { 21.11Scarpellini et al } \\
\text { 21.13Sokolovic } \\
\text { 21.14Zahary e } \\
\text { Thurasamy }\end{array}$ & $\begin{array}{l}21.1 \\
\\
21.3 \\
21.4 \\
21.5 \\
21.6 \\
21.7 \\
21.8 \\
21.9 \\
21.10 \\
21.11 \\
21.13 \\
21.14\end{array}$ & $\begin{array}{l}21.4 \\
21.5 \\
21.6 \\
\\
21.8\end{array}$ & $\begin{array}{l}21.10 \\
21.11 \\
21.14\end{array}$ & $\begin{array}{l}21.1 \\
21.3 \\
21.4 \\
21.5 \\
21.6 \\
21.7 \\
21.8 \\
21.9 \\
21.10 \\
21.11 \\
21.13 \\
21.14\end{array}$ \\
\hline 2013 & $\begin{array}{l}\text { 22.1Tseng } \\
\text { 22.2Siltaoja } \\
\text { 22.3Gavronski } \\
\text { 22.4Jabbour et al } \\
\text { 22.5Hallstedt } \\
\text { 22.6Quist e Tukker } \\
\text { 22.7Vicente-Molina } \\
\text { et al } \\
\text { 22.8Wong } \\
\text { 22.9Keskin et al }\end{array}$ & $\begin{array}{l}22.1 \\
22.2 \\
22.3 \\
22.4 \\
22.5 \\
22.6\end{array}$ & $\begin{array}{l}22.1 \\
22.2 \\
22.3 \\
22.4 \\
22.5 \\
22.6 \\
22.7\end{array}$ & $\begin{array}{l}22.4 \\
22.5 \\
22.6 \\
22.7\end{array}$ & $\begin{array}{l}22.1 \\
22.2 \\
22.3 \\
22.4 \\
22.5 \\
22.6 \\
22.7 \\
\\
22.8 \\
22.9\end{array}$ \\
\hline
\end{tabular}

Tabela 1 - Levantamento bibliográfico dos campos de estudo 
Todo este processo ocorre no âmbito das grandes empresas, as quais possuem um sistema de gestão ambiental (SGA), apoiado pela certificação ISO 14001, que é capaz de impulsionar o processo de inovação, que por fazer uma rigorosa documentação das práticas de gestão ambiental, promove a codificação e a retenção do conhecimento relevante e essencial para o processo, ou seja, a certificação acaba induzindo a aprendizagem e fornecendo informações ambientais (Aravind, 2012; Carruthers, 2012; Kemp e Foxon, 2007; Horbach et al., 2008; Melnyk et al., 2003; Reid e Miedzinski, 2008). A suposição é que o SGA certificado pela ISSO 14001 facilite as ecoinovações diretamente, por introduzir metas ambientais e estruturas de gestão, assim como programas para atingí-las, e indiretamente, por induzir à aprendizagem organizacional e fornecer informações ambientais críticas, ou relevantes (Jabbour et al, 2008; Melnyk et al, 2003; Sokolovic, 2012). Considerando todos estes aspectos, a pesquisa tem seu foco em interações verticais, horizontais e comportamentais que promovam o compartilhamento do conhecimento e seu uso para gerar ecoinovações.

\section{Teorização}

A sustentabilidade requer a integração dos aspectos sociais e ambientais na visão, na cultura e nas operações da empresa, onde um profundo processo de mudança organizacional é essencial (Baumgartner e Zielowski, 2007; Huang e Wu, 2010; Jabbour et al., 2008; Jabbour et al., 2011; Jabbour et al., 2013; Klassen e Whybark, 1999; Noci e Verganti, 1999; Paraschiv et al., 2012; Porter e Van der Linde, 1995; Quist e Tukker, 2013). Para atingir um desempenho sustentável, a organização deve ir além de simples conformidade com as necessidades legislativas, contribuindo ativamente para o desenvolvimento sustentável pela introdução de produtos e serviços inovadores (Falk e Ryan, 2007; Hellstrom, 2007; Horbach et al., 2008; Horbach et al., 2012; Kemp e Foxon, 2007; Kemp e Volpi, 2008; Rennings, 2000; Santolaria et al., 2011). Tais afirmativas motivaram a consecução de um referencial teórico sustentado por estudos em gestão do conhecimento ambiental.

\subsection{Gestão do Conhecimento Ambiental}

A Gestão do Conhecimento Ambiental (GCA) pode ser definida como um "sistema de ligação de dados, análise e pessoas, que apresenta uma oportunidade para formalizar a ecologia industrial em uma empresa" (Wernick, 2003). Wernick (2003) usa empresas na América do Norte em seus relatórios como exemplos de que a GCA melhora o desempenho da empresa através da gestão do conhecimento, e não só pode melhorar a inovação ecológica industrial, mas também reduz o custo da busca de informação.

A GCA pode ser considerada como um poderoso sistema de gestão do conhecimento na área de aprendizagem do conhecimento ambiental, que permite às organizações moverem-se em direção a sustentabilidade (Huang et al., 2009; Huang et al., 2010; Huang e Wu, 2013; Wernick, 2003). Trata-se de uma atividade projetada para decidir sobre a estratégia e implementação das atividades organizacionais, com o objetivo final de melhorar o desempenho financeiro e ambiental.

Um exemplo é processo conhecido como Processo de Circulação do Conhecimento Ambiental (EKCP - Environmental Knowledge Circulation Process), que permite a 
avaliação do desempenho ambiental da empresa, por meio de seus cinco componentes: criação, acúmulo, compartilhamento e internalização do conhecimento ambiental. Tal recurso visa converter o conhecimento ambiental externo em valores internos da empresa. Há a necessidade de estratégias de treinamento neste contexto, desenhadas para motivar e melhorar o conhecimento ambiental dos funcionários e a consciência ambiental (Gavronski et al., 2013; Shrivastava, 1995). A seguir, são expostos os elementos que formam este processo:

Criação de conhecimento ambiental: é o entendimento da necessidade de desenvolver novas tecnologias e sistemas para melhorar a criatividade e competitividade.

Acúmulodeconhecimentoambiental:érepresentadopela experiência experimentação. Considerando uma perspectiva baseada em recursos, o conhecimento tecnológico, as habilidades, as experiências dos funcionários são valiosos ativos que são acumulados ao longo do tempo na empresa e são incorporados na cultura organizacional. Neste estágio, o estoque de conhecimento é encontrado a nível individual, e, portanto, é necessário um contínuo esforço para a aquisição da experiência dos funcionários e alocação dos recursos organizacionais. Neste estágio há uma crescente atividade de P\&D.

Compartilhamento de conhecimento ambiental: é um tipo de transação do conhecimento entre indivíduo e grupo.

Internalização de conhecimento ambiental: é o processo de aprendizagem para converter o conhecimento explícito em conhecimento tácito. Os empregados neste estágio podem acessar as informações ambientais e o conhecimento necessário para completar as tarefas, através de séries de sessões de treinamentos ambientais e exercícios.

O EKCP é responsável por melhorar continuamente a gestão ambiental. A avaliação dos seus componentes inclui a capacidade organizacional para internalizar as tarefas relacionadas ao conhecimento ambiental, as oportunidades de educação ambiental e a aprendizagem organizacional (Lee e Rhee, 2005; Vicente-Molina et al., 2013).

O contexto para o EKCP deve ser envolto pelo Processo de Conversão do Conhecimento (SECI) idealizado por Nonaka e Takeuchi (1995), constituído por quatro estágios:

Socialização: O conhecimento tácito é ganho a partir da experiência acumulada dos indivíduos;

Externalização: Acúmulo de experiência e conhecimento são convertidos em sistemático conhecimento explícito;

Combinação: As opiniões são trocadas entre comunidades do conhecimento e comunidade de prática, internamente e externamente;

Internalização: O conhecimento ambiental é internalizado dentro da ética da empresa e responsabilidade social.

Pode-se observar pelas etapas acima, que o compartilhamento de informações ocorrendo por meio de um efetivo processo de GC é o fundamento para criar e implementar um método de melhoria e desenvolver novas tecnologias e desenho de produtos ambientalmente corretos. 
No 'negócio verde', o conhecimento ambiental é um ativo intangível valioso para criar e sustentar uma vantagem competitiva. A gestão sistemática do conhecimento ambiental e do banco de dados ambiental pode melhorar a cultura de inovação na organização e auxiliar os funcionários no acúmulo das habilidades individuais. O compartilhamento do conhecimento ambiental é imprescindível para uma organização mais ativa, e o processo de circulação do conhecimento ambiental torna-se mais eficaz através da aprendizagem e formação contínua. Tais práticas podem melhorar a consciência ambiental dos funcionários, que irão se beneficiar dos valores ambientais essenciais da empresa (Huang et al., 2009).

Percebeu-se, então, que a GCA é uma atividade ambiental que deve ser contínua, no intuito de auxiliar os gestores a exercerem decisões estratégicas ecoeficientes e a identificar melhores projetos e investimentos em P\&D para alcançar a meta do desenvolvimento sustentável. A seção a seguir traz a relação proposta como discussão, ressaltando-se o comprometimento da alta gestão e o estímulo à criatividade para a criação de conhecimento e a subsequente geração de idéias ecoinovadoras.

\section{Discussão}

Gerir a inovação nas empresas representa na atualidade uma das mais importantes e sustentáveis fontes de vantagens competitivas. Partindo desse ponto de vista, as empresas têm passado a programar estratégias e práticas ambientais pró-ativas usando iniciativas de gestão para mitigar os impactos de suas atividades de inovação no ambiente. O comprometimento da alta gestão, assim como da gestão de nível médio, tem uma significativa influência na prática de uma gestão ambiental bem-sucedida internamente na empresa.

Outro importante fator para realçar o comprometimento da alta gestão, através da educação, treinamento, e profunda intervenção na cultura organizacional, são as dificuldades encontradas na mudança organizacional devido à implementação de sistemas de gestão ambiental, que principalmente derivam da ausência de esforço da empresa em minimizar a resistência dos empregados e a ausência de consciência dos mesmos com relação aos danos que suas atitudes podem causar ao ambiente e a empresa em si (Oliveira e Pinheiro, 2009). Por estes aspectos e especificidades, a gestão deve fomentar a criação de conhecimento, a P\&D, a criatividade e as habilidades dos empregados, os sistemas para proteção tecnológica, explícitos ou tácitos, as curvas de aprendizagem e a prática crítica das rotinas. A partir dessa perspectiva, a primeira proposição é construída:

Primeira Proposição: As diferentes estratégias ou práticas ambientais são fortemente dependentes de interpretações gerenciais, que podem ser vistas tanto como ameaças ou oportunidades para enfrentar as diversas questões relacionadas ao ambiente.

A primeira proposição visa ressaltar o papel dos gestores em aumentar a consciência ambiental, importante quando da implementação das certificações ambientais, como por exemplo, a ISO 14001 e as preocupações ambientais gerenciais estarem positivamente relacionadas ao escopo e a velocidade de resposta às questões ambientais (Tseng et al., 2013). 
Para identificar o que dirige a ecoinovação, de acordo com Tseng et al. (2013), é necessário primeiro definir o que ela representa na produção. As ecoinovações podem ser classificadas em quatro categorias, assim como as inovações tradicionais: inovação da gestão; inovações de processo; inovações de produto e inovações tecnológicas. As empresas têm cada vez mais necessitado considerar a avaliação do ciclo de vida dos produtos para melhorá-los (processo de desenho).

Para Johansson e Magnusson (1998), a ecoinovação pode ser entendida quando novos produtos e processos fornecem valor aos negócios, enquanto fazem uso de menos recursos e resultam na redução dos seus impactos ambientais. Em geral, quando se fala em ecoinovação, estas podem ser formadas conceitualmente como produtos novos, incrementados, processos ambientalmente mais sustentáveis, ou inovações tecnológicas constituídas por componentes de menor impacto ambiental (produtos com eficiência melhorada em comparação a outros produtos existentes, e cuja avaliação do ciclo de vida demonstra claras vantagens).

No entanto, nem sempre uma inovação tecnológica ou até mesmo uma inovação de produto apresentam características positivas, ou seja, é necessária a existência de um método de avaliação para estes aspectos. Por exemplo, a empresa Dow Chemicals criou uma espécie de ferramenta, simples a princípio, o 'Eco-Compass', com a finalidade de avaliar as melhorias ambientais, a qual possui seis dimensões que representam as dimensões ambientais relevantes, sendo elas: saúde e riscos ambientais, conservação dos recursos, intensidade de energia e de materiais, revalorização (remanufatura, reuso, reciclagem) e extensão dos serviços. Essas dimensões são utilizadas para avaliar as inovações de acordo com seus méritos ambientais. As inovações devem ser avaliadas em todas as dimensões para assegurar que o mérito ambiental em uma dimensão não seja contrabalanceado pelo aumento dos impactos ambientais em outra dimensão.

Ainda, esta ferramenta é útil para identificar possibilidades para melhorias ambientais e estimular a criatividade ambiental. Além desse instrumento existem outros, como o LIDS Wheel ou 'Eco-design Strategy Wheel' e a 'Intensidade de Material por unidade de serviço', que podem ser usados de modo semelhante ao Eco-Compass (Johansson e Magnusson, 1998).

Esta “criatividade ambiental”, mencionada anteriormente, deve buscar respaldo em uma prática específica, a gestão do conhecimento (GC). A GC é um processo necessariamente social, provido de estratégias, objetivos e etapas simultâneas, que visa desenvolver nas pessoas a capacidade de percepção, de criação de significado e de construção de conhecimentos. $\mathrm{O}$ conhecimento pertinente à organizações de negócios é composto por fatos, opiniões, idéias, teorias, princípios, modelos, valores, experiências, informações, contexto e intuições. Tem sua origem na mente dos indivíduos e nas organizações aparece embutido não só em documentos e repositórios, mas também em rotinas, processos, práticas e normas organizacionais (Mitri, 2003).

Uma das mais influentes teorias sobre criação de conhecimento organizacional é a defendida por Nonaka e Takeuchi (1995). Em suas análises, a organização cria conhecimento através da conversão e interação entre as dimensões tácito e explícito. A conversão de conhecimento ocorre em quatro modos: do conhecimento tácito para tácito - socialização; do conhecimento tácito para o explícito - externalização; do 
conhecimento explícito para explícito - combinação; e do conhecimento explícito para o tácito - internalização. A Figura 1 traz os quatro modos de conversão bem como suas características principais.
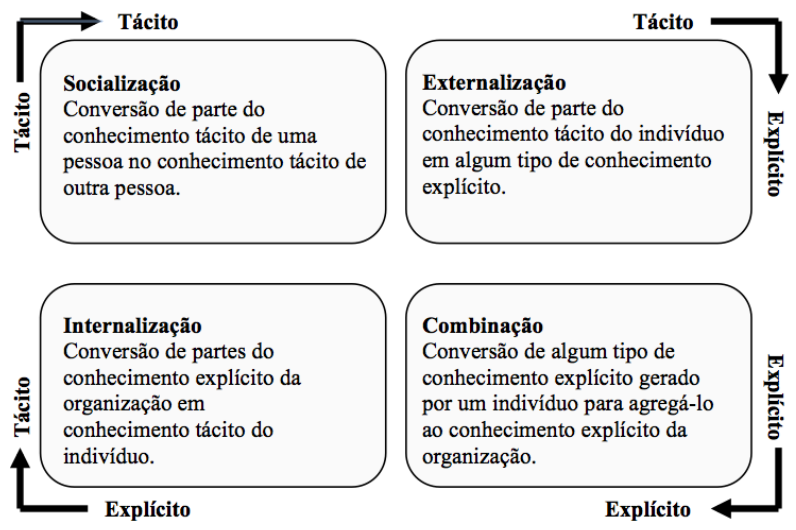

Figura 1 - Modelo SECI de conversões do conhecimento. Fonte: Adaptação dos textos de Nonaka e Takeuchi (1995) e Silva (2002).

Esse processo é entendido como a capacidade da organização para criar conhecimento, difundi-lo em todas as áreas e incorporá-lo a bens, serviços e sistemas (Johannessen et al., 1999). Tendo isso em vista, a segunda proposição é construída:

Segunda proposição: Se houver uma preocupação da gerência com o estímulo e a adoção de ferramentas para a geração de idéias ecoinovadoras, a aprendizagem dos funcionários será respaldada pela gestão do conhecimento.

Nas próximas seções, o artigo estabelece, através de duas metodologias, fortes relacionamentos entre a ecoinovação e a gestão do conhecimento, onde se vislumbra que essa relação pode ser traçada por meio de mapas específicos das atividades ambientais relacionados a aspectos do conhecimento, e até mesmo oferecerem direcionamentos para a obtenção de oportunidades de melhorias ambientais nas empresas.

\subsection{A relação entre ecoinovação e a GC sob a ótica do Standard Design Process Form (SDPF) e do Diagrama de PIT (Product Ideas Tree Diagram)}

Com o aumento da consciência dos consumidores por produtos mais eficientes ambientalmente e devido às pressões das leis ambientais, o desenho de produtos mais sustentáveis (Sustainable Product Design - SPD) vem sendo visto como uma oportunidade para melhorar seus produtos, processos e serviços. Tanto os negócios quanto o mundo acadêmico têm identificado a necessidade para abordagens estratégicas para SPD que resultarão, portanto, em melhorias de mudanças radicais no desenho dos produtos e serviços. O SPD é visto por um ângulo onde ocorre o balanceamento entre as questões ambientais, éticas e sociais no desenho do produto e no seu desenvolvimento. Este conceito engloba duas abordagens diferentes: o ecodesign e a ecoinovação (Jones et al., 2001). 
De acordo com Jones et al. (2001), o ecodesign, apesar de focar em todo o ciclo de vida do produto desde a extração das matérias-primas até o fim de vida e descarte, apresentase como limitado no que se liga a melhorias mais radicais nos produtos, uma vez que é um desenho de uma atividade específica que foca no redesenho ou na otimização dos produtos existentes. Portanto, as mudanças tendem a ser incrementais e o resultado apresenta apenas um percentual de redução dos impactos ambientais globais dos produtos. Tanto o mundo corporativo quanto o mundo acadêmico tem identificado a necessidade para novas abordagens de SPD que resultem em significantes melhorias no desenho dos produtos ou serviços, que podem ser chamadas de não-incrementais ou melhorias de mudança radical. Esse foco nos chama a observar que os fatores e aspectos ambientais devem ser integrados anteriormente ao processo de desenvolvimento de produtos; e é exatamente nesse aspecto, que a ecoinovação se diferencia do ecodesign.

Foi observado que, para todas as inovações, sejam elas tradicionais ou ecoinovações, devem existir formas para avaliar os méritos ambientais de um produto em relação a outro, para que não ocorra de um aspecto positivo ser contrabalanceado por um aspecto negativo, por exemplo. Portanto, como já visto também, existem algumas abordagens ou técnicas, como o Eco-Compass que são capazes de condensar as informações ambientais em um mapa visual que possa comparar os méritos ambientais de opções de novos desenhos contra um desenho original. Tanto o Eco-Compass quanto o LIDS Wheel são ferramentas que podem fornecer pontos iniciais chaves para estruturar sessões de brainstorming para a ecoinovação.

\begin{tabular}{|c|c|c|}
\hline DESENCADEAMENTO & $\begin{array}{l}\text { COLETA DE } \\
\text { INFORMACŌES }\end{array}$ & \\
\hline & SINTESE & \\
\hline & ANÁLISE & \\
\hline$\downarrow$ & $\begin{array}{l}\text { TIPO DE PRODUTO } \\
\text { ESPERADO }\end{array}$ & $\begin{array}{l}\text { ESTRATÉGIA DE } \\
\text { NEGÓCIOS } \\
\text { AMBIENTAL }\end{array}$ \\
\hline $\begin{array}{l}\text { PLANEJAMENTO DO } \\
\text { PRODUTO }\end{array}$ & $\begin{array}{l}\text { COLETA DE } \\
\text { INFORMAÇŌES }\end{array}$ & \\
\hline & SINTESE & \\
\hline & ANÁLISE & \\
\hline & $\begin{array}{l}\text { TIPO DE PRODUTO } \\
\text { ESPERADO }\end{array}$ & $\begin{array}{l}\text { PLANO DE PROJETO } \\
\text { EM ECOINOVACCÃO }\end{array}$ \\
\hline CONCEPÇÁO DO & COLETA DE & \\
\hline DESENHO & $\begin{array}{l}\text { INFORMAÇÕES } \\
\text { SINTESE }\end{array}$ & \\
\hline & ANÁLISE & \\
\hline & $\begin{array}{l}\text { TIPO DE PRODUTO } \\
\text { ESPERADO }\end{array}$ & $\begin{array}{l}\text { CONCEITO DE } \\
\text { DESENHO AMBIENTAL }\end{array}$ \\
\hline INCORPORAÇÃO DO & COLETA DE & \\
\hline DESENHO & $\begin{array}{l}\text { INFORMAÇÕES } \\
\text { SINTESE }\end{array}$ & \\
\hline & ANÁLISE & \\
\hline & $\begin{array}{l}\text { TIPO DE PRODUTO } \\
\text { ESPERADO }\end{array}$ & $\begin{array}{l}\text { IDÉIASS DE PRODUTO } \\
\text { 'VERDE' }\end{array}$ \\
\hline $\begin{array}{l}\text { DETALHËS DO } \\
\text { DESENHO }\end{array}$ & $\begin{array}{l}\text { COLETA DE } \\
\text { INFORMAÇŌES }\end{array}$ & \\
\hline & SÍNTESE & \\
\hline & ANALISE & \\
\hline & $\begin{array}{l}\text { TIIPO DE PRODUTO } \\
\text { ESPERADO }\end{array}$ & $\begin{array}{l}\text { ESPECIFICAÇÃO DO } \\
\text { PRODUTO }\end{array}$ \\
\hline $\begin{array}{l}\text { FABRICAÇÃO E } \\
\text { LANÇAMENTO }\end{array}$ & & \\
\hline
\end{tabular}

Figura 2 - Processo de desenho padrão - SDPF (Baseado na BS 700o). Fonte: Adaptada de Inns (1994). 
Ainda no contexto do SPD, existe uma outra abordagem que trata do processo de geração de idéias dentro da ecoinovação. Esse processo inclui duas ferramentas que foram desenvolvidas, o Standard Design Process Form (SDPF) e o Product Ideas Tree (PIT) diagram que demonstram seu potencial em avaliar e documentar idéias por todo o processo de ecoinovação. Esse processo de documentação e registro é visto como uma melhoria na gestão da ecoinovação por todo o processo de desenho.

O Standard Design Process Form (SDPF) é utilizado para descrever onde a geração de idéias está ocorrendo no processo de desenho e qual o tipo de atividade de desenho está sendo conduzida. O SDPF divide o desenvolvimento de produto em estágios cronológicos, com cada estágio possuindo um diferente ponto inicial e um esperado tipo de produto. Ainda, este processo é uma versão do processo de desenho adaptada para os processos de ecoinovação, onde os tipos de produtos definidos são aqueles tipicamente esperados para os projetos de ecodesign (Jones et al., 2001). A Figura 2 ilustra este conceito:

Ao examinar a idéia do produto a partir dos estudos de caso, poderão ser determinadas as atividades de desenho dominantes. As fases do SDPF podem ser observadas na figura 3 (primeira coluna), sendo compostas pela coleta de informações, síntese, análise e tipo de produto esperado (segunda coluna), e onde cada tipo de produto esperado, por sua vez, possui como foco um aspecto específico (terceira coluna). Existe um feedback e interatividade entre as fases e o tipo de produto esperado.

Percebeu-se que o SDPF pode ser utilizado para identificar onde as ferramentas e metodologias de ecoinovação existentes se adequam no processo de desenvolvimento de produtos. Existem duas etapas neste processo: Na primeira etapa, determina-se o estágio teórico do processo de desenho em que a atividade ocorre (tipo de produto esperado). Na segunda etapa, as atividades de desenho em cada estágio podem ser constituídas da mistura dos três seguintes tipos de atividades de desenho distintas: a união de informações, a síntese (pensamento divergente) e a análise (seleção de idéias).

Terceira Proposição: O processo de desenho padrão é uma ferramenta de estímulo à criação de conhecimento, uma vez que engloba as atividades: 1 - Compartilhamento e criação de conhecimento tácito através da experiência direta; 2 - Articulação do conhecimento tácito através do diálogo e reflexão; 3 - Sistematização e aplicação do conhecimento explícito e informações; 4 - Aprendizagem e aquisição de novos conhecimentos tácitos na prática (Nonaka e Toyama, 2003).

Chegou-se a esta proposição a partir da tentativa de estabelecer um relacionamento entre a fase de Concepção do Desenho do SDPF e o processo SECI de Nonaka e Takeuchi (1995).

A segunda ferramenta, o Diagrama de PIT (Product Ideas Tree), foi criado inicialmente pela necessidade de uma estrutura que possibilitasse a reunião dos resultados obtidos a partir de workshops criativos de ecoinovação. Este diagrama se coloca como uma atividade de registro que se diferencia de um simples registro de idéias ou técnica de mapeamento, uma vez que as idéias são simultaneamente reunidas de acordo com as estratégias 'títulos' (principais), ou as 'idéias chaves' obtidas a partir do LIDS Wheel e Eco-Compass e que são colocadas dentro dos estágios aplicáveis do processo de desenho (Jones et al., 2001). 
É cabível que o Diagrama de PIT seja utilizado como parte integral dos processos de ecoinovação: primeiramente, ao lado das ferramentas de ecoinovação existentes e metodologias como um método de documentação, e secundariamente, visto durante as sessões criativas como ferramenta de registro. A Figura 3 apresenta o Diagrama de PIT:

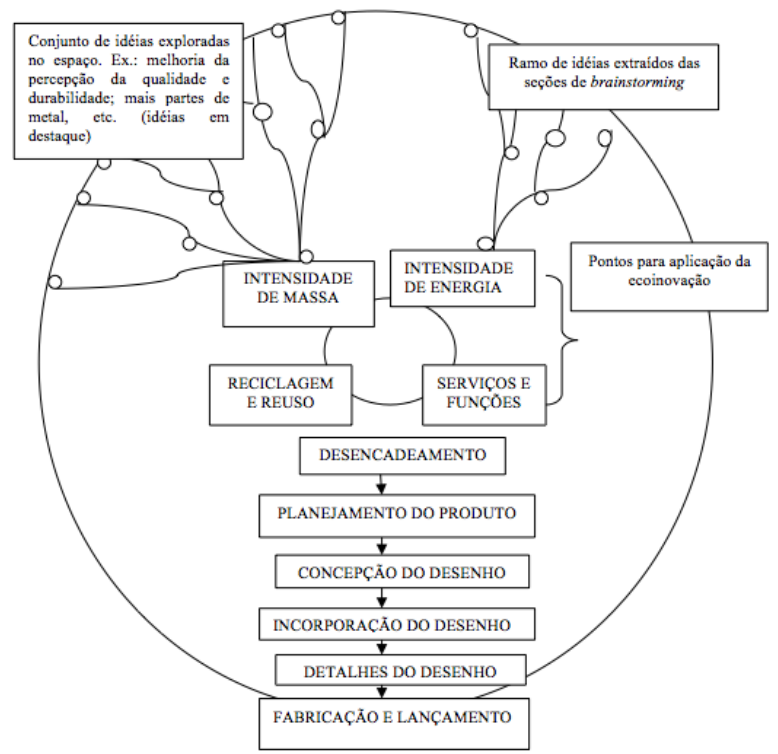

Figura 3 - Diagrama de PIT. Fonte: Adaptado de Jones et al. (2001).

De acordo com o Diagrama de PIT, as idéias são classificadas em consonância com a sua relevância nos estágios do processo de desenho e sua relevância nas estratégias ambientais (tomadas a partir do Eco-Compass e LIDS Wheel). O diagrama mostra a quantidade de idéias registradas, onde cada ponto representa uma idéia; cada conjunto de ramos representa uma sessão de brainstorming durante um workshop. O objetivo deste mapa é a identificação de oportunidades para mais geração de idéias ou identificar um menor número de idéias relevantes, ou seja, é a identificação de oportunidades de melhorias ambientais, no caso, através de sessões de brainstorming em empresas, que são vistas como uma forma eficaz de compartilhamento de conhecimento.

Quarta Proposição: O Diagrama de PIT é mais uma ferramenta que visa suprir a necessidade de se obter um registro das principais idéias advindas das sessões de brainstormings, forma de compartilhamento efetivo de conhecimento, para que se possam registrar as idéias 'chaves', por meio das quais serão obtidas oportunidades de melhorias para pontos específicos envolvidos no núcleo ambiental da empresa, como: massa, energia, reciclagem e reuso.

Existe uma outra ferramenta, conhecida como metodologia STRECHT (Jones et al., 2001) adotada pela Philips Sound and Vision, que confirma a importância de se focar nos estágios anteriores do processo de desenho, de planejar o produto. Essa 
metodologia foca na incorporação dos aspectos ambientais na estratégia de negócios da companhia; antecipa as futuras oportunidades ambientais e ameaças nas fases anteriores do processo de desenho e foca na obtenção da ecoeficiência ao invés de melhorias incrementais ambientais.

Vale ressaltar que a proposta desse estudo foi de lançar luz sobre a relevância de métodos e ferramentas que compõem as etapas iniciais de desenho de produtos inovadores e mais sustentáveis. Tais ferramentas fazem parte do início do processo ecoinovador, o desenho do produto. Acredita-se que as maiores oportunidades de aprendizagem estão nesse estágio, se fomentado pela gestão do conhecimento. Por fim, as ferramentas apresentadas nesta seção diferem-se da maioria dos estudos encontrados na literatura, acerca de métodos desenvolvidos apenas para o estágio de avaliação de impactos ambientais.

\section{Considerações finais}

O artigo procurou, através das constatações e das proposições teóricas que são contribuições deste trabalho, ressaltar a importância e os benefícios que a Gestão do Conhecimento pode trazer para a área de Ecoinovação. Como exemplo de tais benefícios, discutiu-se, mais especificamente, sobre o estágio de desenho de produtos ecoinovadores.

O SDPF foi abordado como forma de ilustrar uma rica ferramenta na qual a criação de conhecimento é beneficiada, uma vez que esse processo de desenho descreve onde a geração de idéias está ocorrendo e qual o tipo de atividade de desenho está sendo conduzida.

Percebeu-se ainda que o Diagrama de PIT pode ser considerado outra ferramenta de apoio à GC, pois auxilia no mapeamento, registro, reunião e seleção das melhores e principais idéias advindas de sessões de brainstorming nas empresas com a finalidade de colaboração na busca por oportunidades de melhorias ambientais focando no redesenho dos produtos.

As quatro proposições construídas durante as seções do artigo revelaram algumas respostas para a questão de pesquisa, em especial no que se refere às fases de conversão dos conhecimentos tácito e explícito, que por fim resultam no processo de criação que dará origem à ecoinovação.

Uma das vertentes principais em que pensamos a princípio quando levamos em conta as questões ambientais é o contexto social. Em geral, quando falamos de mudanças nos padrões de consumo para se obter uma sociedade mais sustentável, com maior preocupação voltada para a redução dos impactos no ambiente, pensa-se instantaneamente no contexto social, ou melhor, nas conseqüências que os padrões industriais acarretarão neste contexto. Deste modo, futuras pesquisas poderiam concentrar-se em analisar os impactos que estas metodologias e técnicas voltadas à ecoinovação geram no âmbito social, que possibilitam uma aprendizagem por meio dos processos de conhecimento.

Outrossim, dado o caráter inovador no relacionamento entre a ecoinovação e a gestão do conhecimento, uma outra consideração para futuros estudos seria a identificação 
de fatores críticos e novas ferramentas-suporte para ambas as práticas, no intuito de beneficiar empresas ecoinovadoras.

\section{Referências}

AMABILE, T. M. (1998). How to kill creativity. Harvard Business Review, 76 (5), 76-87.

ANDERSEN, M. M. (2008). Eco-innovation: towards a taxonomy and a theory. In Proceedings of the Druid Society Conference 2008, Entrepreneurship and innovation, (17-20), London.

ARAVIND, D. (2012). Learning and innovation in the context of process-focused management practices: The case of an environmental management system. Journal of Engineering and Technology Management, 29(3), 415-433.

BAUMGARTNER, R.J., \& ZIELOWSKI, C. (2007). Analyzing zero emission strategies regarding impact on organizational culture and contribution to sustainable development. Journal of Cleaner Production, 15, 1321-1327.

BLOODGOOD, M. J. (2012). Performance implications of matching adaption and innovation cognitive style with explicit and tacit knowledge resources. Knowledge Management Research \& Practice, 10, 106-117.

BOIRAL, O. (2002). Tacit knowledge and environment management. Long Range Plann, 35(3), 291-317.

BLOMQUIST, T., \& SANDSTROM, J. (2004). From issues to checkpoints and back: managing green issues in R\&D. Business Strategy and the Environment, 13(6), $363-373$.

BRANNBACK, M. (2003). R\&D collaboration: role of $\mathrm{Ba}$ in knowledge-creating networks. Knowledge Management Research \& Practice, 1, 28-38.

CARRILLO-HERMOSILLA, J., DEL RÌO, P., \& KONNOLA, T. (2012). Policy Strategies to promote Eco-innovation. Journal of Industrial Ecology, 14(4), 541-557.

CARRUTHERS, G., \& VANCLAY, F. (2012). The intrinsic features of Environmental Management Systems that facilitate adoption and encourage innovation in primary industries. Journal of environmental management, 110, 125-34.

COHEN, W.M., \& LEVINTHAL, D.A. (1990). Absorptive capacity: a new perspective on learning and innovation. Administrative Science Quaterly, 35(1), 128-152.

DANGELICO, R. M., \& PUJARI, D. (2010). Mainstreaming green product innovation: why and how companies integrate environmental sustainability. Journal of Business Ethics, 95(3), 471-486.

DORAN, J., \& RYAN, G. (2012). Regulation and firm perception, eco-innovation and firm performance. European Journal of Innovation Management, 15, 421-441.

ELMQUIST, M., \& SEGRESTIN, B. (2009). Sustainable development through innovative design: lessons from the KCP method experimented with an automotive firm. International Journal of Automotive Technology and Management, 9(2), 229-244. 
EL SAWY, O.A, ERIKSSON, I., \& RAVEN, A. (2001). Understanding shared knowledge creation spaces around business processes: precursors to process innovation implementation. International Journal of Technology Management, 22(1-3), 149-173.

FALK, J., \& RYAN, C. (2007). Inventing a sustainable future: Australia and the challenge of eco-innovation. Futures, 39(2-3), 215-229.

FREIXO, J., \& ROCHA, Á. (2014). Arquitetura de informação de suporte à gestão da qualidade em unidades hospitalares. RISTI - Revista Ibérica de Sistemas e Tecnologias de Informação, (14), 1-15.

GAVRONSKI, I., KLASSEN, R. D., VACHON, S., \& NASCIMENTO, L. F. M. (2012). A learning and knowledge approach to sustainable operations. International Journal of Production Economics, 140(1), 183-192.

GAVRONSKI, I., PAIVA, E.L., TEIXEIRA, R., \& ANDRADE M.C.F. (2013). ISO 14001 certified plants in Brazil- taxonomy and practices. Journal of Cleaner Production, $39,32-41$.

HALLSTEDT, S.I., THOMPSON, A.W., \& LINDAHL, P. (2013). Key elements for implementing a strategic sustainability perspective in the product innovation process. Journal of Cleaner Production, 51, 277-288.

HART, S.L. (1995). A natural-resource-based view of the firm. Academy of Management Review, 20(4), 986-1014.

HELLSTROM, T. (2007). Dimensions of Environmentally Sustainable innovation: the Structure of Eco-innovation concepts. Sustainable Development, 15(3), 148-159.

HORBACH, J. (2008). Determinants of environmental innovation: New evidence from German panel data sources. Research Policy, 37(1), 63-173.

HORBACH, J., RAMMER, C., \& RENNINGS, K. (2012). Determinants of eco-innovations by type of environmental impact: The role of regulatory push/pull, technology push and market pull. Ecological Economics, 78, 112-122.

HUANG, P.S., \& SHIH, L. (2009). Effective environmental management through environmental knowledge management. International Journal of Environmental Science and Technology. 6(1), 35-50.

HUANG, P.S., \& SHIH, L. (2010). The impact of industrial knowledge management and environmental strategy on corporate performance of iso-1400o companies in Taiwan: The application of structural equation modeling. African Journal of Business Management, 4(1), 21-30.

HUANG, Y.C., \& WU, Y.C.J. (2010). The effects of organizational factors on green new product success: Evidence from high-tech industries in Taiwan. Management Decision, 48(10), 1539-1567.

IANSITI, M., \& CLARK, K. (1994). Integration and dynamic capability: evidence from product development in automobiles and mainframe computers. Industrial and Corporate Change, 33(3), 557-605. 
INKPEN, A. C. (1996). Creating knowledge through collaboration. California Management Review, 39(1), 123-140.

INNS, T. (1994). BS 70oo and the management design of the design process in course document. London: Design Research Centre, Brunel University.

JABBOUR, C.J.C., \& SANTOS, F.C.A. (2008). Relationships between human resource dimensions and environmental management in companies: proposal of a model. Journal of Cleaner Production, 16(1), 51-58.

JABBOUR, C.J.C., OLIVEIRA, S.V.W.B., \& CASTRO, R. (2011). Cultura Organizacional, Inovação e Gestão Ambiental: integrando conceitos para a edificação de organizações sustentáveis. Revista de Engenharia e Tecnologia, 3(3), 1-10.

JABBOUR, C.J.C., SANTOS, F.C.A., FONSECA, S.A., \& NAGANO, M.S. (2013). Green teams: understanding their roles in the environmental management of companies located in Brazil. Journal of Cleaner Production, 46, 58-66.

JOHANNESSEN, J., OLSEN B., \& OLAISEN, J. (1999). Aspects of innovation theory based on knowledge-management. International Journal of Information, (19), 121-139.

JOHANSSON, G., \& MAGNUSSON, T. (1998). Eco-innovation: a novel phenomenon? The Journal of Sustainable Product Design, (7), 7-15.

JONES, E., HARRISON, D., \& MCLAREN, J. (2001). Managing Creative Eco-innovation. Structuring outputs from Eco-innovation projects. The Journal of Sustainable Product Design, 1(1), 27-39.

JONES, E., STANTON, N.A., \& HARRISON, D. (2001). Applying structured methods to Eco-innovation. An evaluation of the Product Ideas Tree diagram. Design Studies, 22, 519-542.

KAMMERER, D. (2009). The effects of customer benefit and regulation on environmental product innovation: empirical evidence from appliance manufacturers in Germany. Ecological Economics, 68(8-9), 2285-2295.

KEMP, R., \& FOXON, T. (2007). Eco-innovation from an innovation dynamics perspective. Proyecto Measuring Eco-innovation (MEI).

KEMP, R., \& VOLPI, M. (2008). The diffusion of clean technologies: a review with suggestions for future diffusion analysis. Journal of Cleaner Production, 16(1), S14-S2.

KESIDOU, E., \& DEMIREL, P. (2012). On the drivers of eco-innovations: empirical evidence from the UK. Research Policy, 41(5), 862-870.

KESKIN, D., DIEHL, J.C., \& MOLENAAR, N. (2013). Innovation process of new ventures driven by sustainability. Journal of Cleaner Production, 45, 50-60.

KLASSEN, R.D., \& WHYBARK, D.C. (1999). Environmental Management in Operations: The Selection of Environmental Technologies. Decision Sciences, 30(3), 601-631. 
KLEEF, V.J.A.G., \& ROOME, N.J. (2007). Developing capabilities and competence for sustainable business management as innovation: a research agenda. Journal of Cleaner Production, 15, 38-51.

KOBAYASCHI, H. (2006). A systematic approach to eco-innovative product design based on life cycle planning. Advanced Engineering Informatics, 20(2), 113-125.

KOBAYASCHI, H., KATO, M., MAEZAWA, Y., \& SANO, K. (2011). An R\&D management framework: for eco-technology. Sustainability, 3(8), 1282-1301.

KROGH, V.G. (1998). Care in knowledge creation. California Management Review, $40(3), 133-154$.

LEE, S.Y., \& RHEE, S.K. (2005). From end-of-pipe technology towards pollution preventive approach: the evolution of corporate environmentalism in Korea. Journal of Cleaner Production, 13(4), 387-395.

LENOX, M., \& EHRENFELD, J. (1997). Organizing for effective environmental design. Business Strategy and Environment, 6(4), 187-196.

LEONARD, D., \& SENSIPER, S. (1998). The role of tacit knowledge in group innovation, California management review 40(3), 112-132.

MALMBORG, V.F. (2007). Stimulating learning and innovation in networks for regional sustainable development: the role of local authorities. Journal of Cleaner Production, 15(17), 1730-1741.

MELNYK, S.A, SROUFE, R.P., \& CALANTONE, R. (2003). Assessing the Impact of Environmental Management Systems on Corporate and Environmental Performance. Journal of Operations Management, 21(3), 329-351.

MITRI, M. (2003). A knowledge management framework for curriculum assessment. Journal of Computer Information Systems, 43(4), 15-24.

NOCI, G., \& VERGANTI, R. (1999). Managing-green product innovation in small firms. R\&D Management, 29(1), 3-15.

NONAKA, I., \& TAKEUCHI, H. (1995). The knowledge creating company: how the Japanese companies create the dynamics of innovation. New York: Oxford University Press.

NONAKA, I., TOYAMA, R., \& KONNO, N. (2000). SECI, Ba and leadership: a unified model of dynamic knowledge creation. Long Range Planning, 33(1), 5-34.

NONAKA, I., \& TOYAMA, R. (2003). The Knowledge-creating theory revisited: knowledge creation as a synthesizing process. Knowledge Management Research \& Practice, $1,2-10$.

OLIVEIRA, O. J., \& SERRA PINHEIRO, C. R. M. (2009). Best practices for the implantation of ISO 14001 norms: a study of change management in two industrial companies in the Midwest region of the state of São Paulo - Brazil. Journal of Cleaner Production, 17(9), 883-885. 
PARASCHIV, D. M., NEMOIANU, E.L., LANGA, C.A., \& SZABÓ, T. (2012). Ecoinnovation, Responsible leadership and organizational change for corporate sustainability. The Amfiteatru Economic, 14(32), 404-419.

PORTER, M.E., \& VAN DER LINDE, C. (1995). Toward a new conception of the environment-competitiveness relationship. The Journal of Economic Perspectives, 9(4), 97-118.

PRAHALAD, C.K., \& HAMEL, G. (1990). The core competence of the corporation. Harvard Business Review, 68(3), 79-93.

PUJARI, D. (2006). Eco-innovation and new product development: understanding the influences on market performance. Technovation, 26(1), 76-85.

QUIST, J., \& TUKKER, A. (2013). Knowledge collaboration and learning for sustainable innovation and comsumption: introduction to the ERSCP portion this special volume. Journal of Cleaner Production, 48, 167-175.

REID, A., \& MIEDZINSKI, M. (2008). Eco-innovation: final report for sectoral innovation watch. Brighton: Technopolis Group.

RENNINGS, K. (2000). Redefining innovation: eco-innovation research and the contribution from ecological economics. Ecological Economics, 32(2), 319-332.

SANTOLARIA, M., OLIVER-SOLÀ, J., GASOL, C.M., MORALES-PINZÓN, T., \& RIERADEVALL, J. (2011). Eco-design in innovation driven companies: perception, predictions and the main drivers of integration: The Spanish example. Journal of Cleaner Production, 19(12), 1315-1323.

SCARPELLINI, S.; ARANDA, A.; ARANDA, J.; LlERA, E.; MARCO, M. R\&D and Eco-innovation: opportunities for closer collaboration between universities and companies through technology centers. Clean Technologies and Environmental Policy, 14(6, 1047-1058, 2012.

SEGARRA-OÑA, M.V., ANGEL, S.P., PASTOR, M., GASPAR, L., \& GARRIGÓS, A.J. (2011). Eco-innovación: una evolución de la innovación? Analísis empírico em la industria cerámica espanhola. Boletín de la Sociedad Española de Cerámica y Vidrio, 50(5), 253-260.

SHRIVASTAVA, P. (1995). Environmental technologies and competitive advantage. Strategic Management Journal, 16(1), 183-200.

SIEBENHUNER, B., \& ARNOLD, M. (2007). Organizational learning to manage sustainable development. Business Strategy and the Environment, 16(5), 339-353.

SILTAOJA, M. E. (2013). Revising the Corporate Social Performance model- Towards Knowledge Creation for Sustainable Development. Business Strategy and the environment. In Press.

SILVA, S. L. (2002). Informação e competitividade: a contextualização da gestão do conhecimento nos processos organizacionais. Ciência da Informação, 31(2), 1 42-151. 
SMITH, M. T. (2001). Eco-innovation and market transformation. The Journal of Sustainable Product Design, 1(1), 19-26.

SOKOLOVIĆ, S. M. (2012). Sustainable Development, Clean Technology. Thermal Science, 16(1), S131-S139.

TIDD, J. (2001). Innovation Management in context: environment, organization and performance. International Journal of Management Reviews, 3(3), 169-183.

TSENG, M.L. (2010). An assessment of cause and effect decision-making model for firm environmental knowledge management capacities in uncertainty. Environmental Monitoring and Assessment, 161(1-4), 549-564.

TSENG, M.L., WANG, R., CHIU, A.S.F., GENG, Y., \& LIN, Y. H. (2013). Improving performance of green innovation practices under uncertainty. Journal of Cleaner Production, 40, 71-82.

VICENTE-MOLINA, M.A., FERNÁNDEZ-SÁINZ, A., \& IZAGIRRE-OLAIZOLA, J. (2013). Environmental knowledge and other variables affecting pro-environmental behavior: comparison of university students from emerging and advanced countries. Journal of Cleaner Production, 61, 130-138.

WERNICK, I. K. (2003). Environmental knowledge management. Journal of Industrial Ecology, 6(2), 7-9.

WONG, S.K.S. (2013). Environmental Requirements, knowledge sharing and green innovation: Empirical evidence from the electronics industry in China. Business Strategy and the Environment, 22(5), 321-338.

ZAHARI, F., \&THURASAMY,R.(2012). Linking Green Product Innovation, Technological and Human Resource capabilities: A conceptual model. In Proceedings of the International Conference on Innovation, Management and Technology Research (ICIMTR2012), Malacca, Malaysia, (21-22). 